\title{
Gas lift optimization in the oil and gas production process: a review of production challenges and optimization strategies
}

\author{
Ikenna Tobechukwu Okorochaa ${ }^{a}$ Chuka Emmanuel Chinwukoa , Chika Edith Mgbemena ${ }^{a}$, \\ Chinedum Ogonna Mgbemena ${ }^{\mathrm{b}^{*}}$ \\ ${ }^{a}$ Department of Industrial/Production Engineering, Nnamdi Azikiwe University, Awka, Nigeria \\ ${ }^{b}$ Department of Mechanical Engineering, Federal University of Petroleum Resources, Efurun, Nigeria \\ *Corresponding Author: mgbemena.ogonna@fupre.edu.ng
}

\section{ARTICLE INFO}

Keywords

Gas Lift;

Optimization;

Oil;

Production Strategies.

\section{Article history}

Received:

July 7, 2020

Revised:

July 14, 2020

Accepted:

July 14,2020

Available online:

21 August, 2020

\section{ABSTRACT}

Gas lift operation involves the injection of compressed gas into a low producing or non-performing well to maximize oil production. The oil produced from a gas lift well is a function of the gas injection rate. The optimal gas injection rate is achieved by optimization. However, the gas lift, which is an artificial lift process, has some drawbacks such as the deterioration of the oil well, incorrect production metering, instability of the gas compressor, and over injection of gas. This paper discusses the various optimization techniques for the gas lift in the Oil and Gas production process. A systematic literature search was conducted on four databases, namely Google Scholar, Scopus, IEE Explore and DOAJ, to identify papers that focused on Gas lift optimizations. The materials for this review were collected primarily via database searches. The major challenges associated with gas lift were identified, and the different optimization strategies available in the literature reviewed. The strategies reviewed were found to be based on artificial intelligence (Al) and machine learning (ML). The implementation of any of the optimization strategies for the gas lift will enhance profitability, reduce operational cost, and extend the life of the wells.

\section{Introduction}

Oil production from production wells decreases gradually with time as the well matures. Mature oil wells across the globe at present accounts for approximately $70 \%$ of oil and gas production (Ismail \& Trjangganung, 2014). Oil production from depleted reservoirs with low energy requires artificial assistance to lift oil from the bottom to the surface. The Sucker rod pump, electric submersible pump, and gas lift are the most common artificial lift methods used in lowering the bottom hole pressure and providing the lift energy to raise the fluids to the surface (Abdalsadig et al., 2016).

The introduction of gas lift to a non-producing or low producing well is a standard method of artificial lift. Figure 1 is a depiction of the gas lift well schematics (Rashid et al., 2012).

The gas lift method involves the injection of compressed gas at extremely high pressure in the annulus, and this softens the fluid column by minimizing their pressure losses and density. The existence of gas inside the production tubing at the deepest point reduces the flow pressure of 
the bottom-hole to allow fluid to flow from the reservoir to the surface (Abdalsadig et al., 2016; Sylvester, 2015).

The gas lift has an adverse effect due to increasing friction losses and, for high injection flow rates, the frictional pressure drops increase tending to be higher than the hydrostatic reduction. Therefore, increasing the gas lift injection rate from a point named technical optimum, which maximize the oil flow rate, implies the reduction of production.

The artificial lift systems are regularly used in the dewatering of gas wells if there is a need to sustain production (Sylvester, 2015). Several parameters are involved in a successful gas lift operation. These parameters, when fully specified, will enhance the production and maximize the operations' net present value (NPV). The most critical parameter in a gas lift operation is the optimum injection rate. Finding the optimum rate is essential because injecting an extra amount of gas not only does not improve production but can decrease it through increased slippage between liquid and gas (Yakoot et al., 2014).

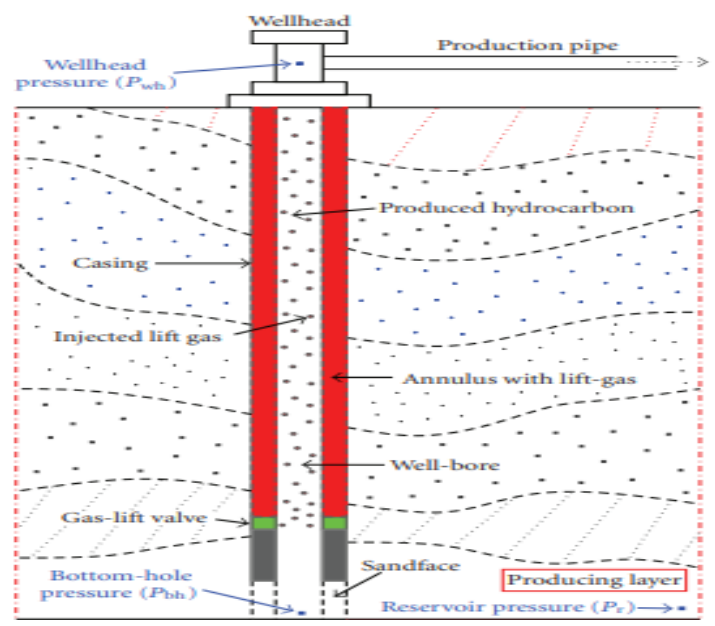

Figure 1. Gas Lift Well Schematic (Rashid et al., 2012)

\section{Literature Review}

Oil production increases typically as gas injection increases. However, too much gas injection will cause undersupply, where the gas phase moves faster than liquid (Saepudin et al., 2007; Sukarno et al., 2009). This condition will ultimately lead to a reduction in oil production. They are identifying optimal gas injection that maximizing oil production is the main interest in gas lift optimization problem.

Some other foreseeable challenges of Gas lift include the worsening of the surface equipment and the sub-surface well completion condition in a mature field, such as deteriorating well integrity with leaks and holes, incorrect production metering, instability of gas compressor availability and efficiency. All those, as mentioned earlier, have resulted in the ineffective gas lift distribution and injection downhole (Ismail \& Trjangganung, 2014). In other to determine the optimal gas lift rate, the usual practice by several operators is to allocate the lift gas to a well according to a gas-lift performance curve(Sylvester, 2015)

The other problems hindering effective gas lifting includes solid/sand handling ability, corrosion/scale handling ability, the stability, number of wells, flowing pressure and temperature limitation, well depth, production rate, flexibility, high Gas-Oil Ratio, electrical 
power, space, economics, etc. (Sylvester, 2015). These factors should be considered before the installation of any of the artificial lift techniques.

The instability phenomenon is one of the critical problems associated with gas allocation optimization. These instabilities affect production drastically and destroy downhole and surface facilities (Mahdiani \& Khamehchi, 2015). Therefore, this study presents an overview of the different optimization methods for production optimization on the gas lift system.

\section{Methodology}

This paper presents a review of the Gas lift optimization techniques employed in the Oil and Gas Production and the production challenges relating to the gas lift process. The method adopted in this review is presented in this section.

\subsection{Search strategy}

A systematic search was conducted to identify papers that focused on Gas lift optimizations. The literature for this study was collected mainly through database searches. The searches were conducted on four databases that included Google Scholar, Scopus, IEE Explore, and DOAJ. The criteria for selection and inclusion for the papers are that they must be written in the English Language must be published from 2007 to 2020. Again, only documents that answered any/or all the following questions were considered eligible.

- Does it involve any Optimization Techniques?

- Does it involve the use of either Artificial Intelligence or Machine Learning?

- Does it attempt to solve any problem experienced by the industry operators in Gas lift process? this review.

Papers published before 2007 and not written in the English Language were excluded in

\section{Results and Discussion}

Optimization problems are real-world problems encountered in many fields of study, such as mathematics, engineering, science, business, and economics. In optimization problems, the target is to find the optimal or most efficient way of using limited resources to achieve the objective of the situation. It may be maximizing the production, minimizing the injected fluid, minimizing the total distance traveled, or reducing the total time to complete a project (Oloro \& Ogbolu, 2013).

The gas available for injection is usually minimal and should be assigned in optimal form for each well. In other to effectively allocate the available gas, an appropriate gas lift optimization method is required. The overriding question is: How do you share the injected gas to each well to yield maximum total oil production rate? In this section, the various methods of gas lift optimization available in the literature are thoroughly discussed.

\subsection{Genetic Algorithm in Gas Lift Optimization}

The Genetic algorithm (GA) is one of the most important meta-heuristic algorithms which was first introduced by Holland in 1975 (Bordbar et al., 2020; Kesavan et al., 2020). It is a type of evolutionary algorithm, which is commonly used in artificial intelligence (Al) and computing (López et al., 2019).

The genetic algorithm (GA) is a stochastic population-based global search procedure in which an initial set of candidate seeds, often unevenly selected, is evolved over several generations using the primary operations of crossover, reproduction, and mutation. The most suitable candidate in the final gene pool is the solution to the optimization problem. The method 
is robust, free from derivative, and can potentially find a global solution. Also, both the integer and continuous variables can be handled with ease.

However, the GA method has the disadvantage of requiring a significant number of function evaluations, and this can be a computationally expensive exercise. Many variations and extensions exist which are used to speed up convergence, maintain population diversity and to avoid binary string encoding (Mahdiani \& Khamehchi, 2015; Rashid et al., 2012; Tavakoli et al., 2017).

\subsection{Least Square Method and Solver in Gas Lift Optimization}

The least-square (LS) method was employed to optimize the gas lift. In a study, the model was tested for reliability, and it was found to be reliable for prediction. Their study was limited to the development of a model using a 10-year production data (Oloro \& Ogbolu, 2013).

The least-square method provides an efficient and unified solution strategy for ensemblebased multi-objective optimization problems. The LS method uses a priori characterization of desirable trade-offs that allows the technique to focus on a single Pareto optimal point (Christiansen et al., 2018). The essential advantage of the LS method is that it reduces the computational complexities compared to other multi-objective optimization techniques.

\subsection{Particle Swarm Optimization Algorithm in Gas Lift Optimization}

The particle swarm optimization (PSO) algorithm is a meta-heuristic computational method that imitates the social behavior of animal swarms. PSO optimizes a problem by improving the candidate solution iteratively. Kennedy and Eberhart first introduced the algorithm in 1995 (López et al., 2019). In the PSO algorithm, two main parameters are being updated in each iteration: the velocity term and position term. The particle's velocity and position are updated through the following equations, respectively:

$v_{i}(t+1)=w v_{i}(t)+c_{1} r_{1}\left(y_{i}(t)-x_{i}(t)\right)+c_{2} r_{2}\left(\hat{y}(t)-x_{i}(t)\right)$

$x_{i}(t+1)=x_{i}(t)+v_{i}(t+1)$

$x_{i}(t) \in U\left[x_{\text {min }}, x_{\text {max }}\right]$

Where $v_{i}(t)$ and $x_{i}(t)$ denotes the velocity and position of particles at time t. $y$ and parameters represent the personal best solution of the particle and global best solution, respectively. $r_{1}$ and $r_{2}$ are the random vectors with uniform distribution in the $[0,1]$ interval. $w, c_{1}$, and $c_{2}$ are the inertia coefficient, personal learning coefficient, and collective learning coefficient, respectively.

$y_{i}(t+1)=y_{i}(t)$ for $\left.f x_{i}(t+1)\right) \geq f y_{i}(t)$

$y_{i}(t+1)=x_{i}(t+1)$ for $\left.f x_{i}(t+1)\right) \leq f y_{i}(t)$

$\left.\left.\hat{y}(t)=y_{o}, y_{1}, \ldots y_{z}=\min f y_{o}(t), f y_{i}(t)\right), \ldots . f y_{z}(t)\right)$

The PSO algorithm is as follows:

$>$ For each particle $i \in 1, \ldots s$, initialise the th position $x_{i}$ and velocity $v_{i}$ randomly.

$>$ Set $y_{i}=x_{i}$

$>\quad$ Evaluate the fitness function, $f\left(x_{i}\right)$ for each value of $i$

$>\quad$ Update $y_{i}$ and $\hat{y}_{i}$ for each value of $i$ (From Equation (3))

$>$ For each dimension $j \in 1 \ldots N_{d}$; update the velocity from

$$
v_{i j}(t+1)=w v_{i j}(t)+c_{i} r_{1 j} y_{i j}(t)-x_{i j}(t)+c_{2} r_{2 j} \hat{y}_{j}(t)-x_{i j}(t)
$$

$>\quad$ Stop the algorithm when the convergence criteria are achieved, otherwise go to step 3.

The particle swarm optimization algorithm has major drawbacks and demerits. The PSO is faster compared to similar evolutionary algorithms. However, its convergence rate does not 
enhance with a higher number of iterations. The main reason is that in the PSO algorithm, particles converge to the point with the personal best and global best solution. To resolve this drawback, the inertia weight $\mathrm{w}$ is used to modify the algorithm. There is also another major drawback reported in the PSO algorithm, in which the quality of solutions is very much dependent on the weighting coefficients and algorithm parameters.

In a reported study, the PSO algorithm was employed to assign an optimum gas injection rate to five oil wells in Iran. A new gas lift performance curve-fit that can minimize the time and volume of the computation was recommended. The algorithm was applied to the five wells in an Iranian oil field (Hamedi et al., 2011).

\subsection{Continuous Ant Colony Optimization Algorithm in Gas Lift Optimization}

The Ant Colony Optimization (ACO) is a new approach to solving discrete and continuous optimization problems which are cost-effective and very fast (Kadkhodaie-llkhchi, 2015). The Ant Colony Optimization (ACO) is a pattern for designing metaheuristic algorithms based on real ants' behavior. These ants deposit pheromones on the ground to mark some favorable pathway that should be followed by other members of the colony (Vijayalakshmi et al., 2015). The ACO exploits a similar mechanism for solving optimization problems.

The Ant Colony Optimization (ACO) is a pattern for designing metaheuristic algorithms based on real ants' behavior. These ants deposit pheromones on the ground to mark some favourable pathway that should be followed by other members of the colony (Duran Toksarı, 2007; Kadkhodaie-Ilkhchi, 2015; Vijayalakshmi et al., 2015; Zerafat et al., 2009). The ACO exploits a similar mechanism for solving optimization problems.

A continuous ant colony optimization algorithm was utilized to allocate the optimum amount of gas to a group of wells for three fields with a different number of wells. The continuous ant colony optimization algorithm shows better gas allocation to the wells in comparison with the previous works with other optimization methods (Ghaedi et al., 2014; Namdar, 2019).

\subsection{Gas Lift Optimization by Real-Time Optimization method}

Real-time optimization (RTO) methods use measurements to offset the effect of uncertainty and drive the plant to optimality. Explicit RTO schemes solve a static optimization problem iteratively, with each iteration requiring the transient operation of the plant to steady-state. However, the implicit RTO methods use transient measurements to bring the plant to steady-state optimality in a single iteration, provided the set of active constraints is known (François \& Bonvin, 2014).

A study on Gas-lift Optimization by Controlling Marginal Gas-Oil Ratio using Transient Measurements was performed. In their study, a novel method was proposed to estimate the marginal GOR online using a dynamic model and transient measurements, without the need for additional perturbation. The proposed method is based on linearizing the dynamic model around the current operating point to estimate the marginal GOR, which is then controlled using simple feedback controllers to achieve optimal operation. In case of disturbances, the proposed method can adjust fast to the new optimal point, without the need to solve computationally expensive optimization problems. By using transient measurements, it does not need to wait for the process to reach a steady-state to update the model. The proposed method was tested in simulations and was shown to provide similar performance as an economic MPC (Krishnamoorthy et al., 2018). 


\subsection{Novel Grey Model (GM) for Gas Lift Optimization}

The Grey model, abbreviated as GM, has been successfully applied in many disciplines (Hsu \& Chen, 2003; Kumar et al., 2018; Ma \& Wu, 2010; Truong \& Ahn, 2012). Grey Model cannot be applied to many real situations and systems. Hence, some scholars have proposed some improvements to GM. The Time-Delayed Polynomial Grey Model (TDPGM) is one of such models proposed by some scholars. The TDPGM is one of the models with good predictive applicability. The Time-Delayed Polynomial Fractional Grey Model (TDPFOGM) was proposed based on the TDPGM model (Chen et al., 2019). The Time-Delayed Polynomial Fractional Grey Model (TDPFOGM) is mathematically represented as:

$x^{\left(\frac{p}{q}\right)}(k)-x^{\left(\frac{p}{q}\right)}(k-1)+\lambda_{1} z^{\left(\frac{p}{q}\right)}(k)=\lambda_{2} \sum_{\tau=1}^{k} \tau^{2}+\lambda_{3} \sum_{\tau=1}^{k} \tau+\lambda_{4}$

Where

$z^{\left(\frac{p}{q}\right)}(k)=\frac{x^{\left(\frac{p}{q}\right)}(k)+x^{\left(\frac{p}{q}\right)}(k+1)}{2}, k=1,2, \ldots, n-1$

The discrete response function can be obtained as

$\hat{x}^{\left(\frac{p}{q}\right)}(k)=x^{(0)}(1) e^{-\lambda_{1}(k-1)}+\sum_{t=2}^{k} \frac{1}{2}\left[e^{-\lambda_{1}(k-t)} f(t)\right]+e^{-\lambda_{1}(k-t)} f(t-1)$

Where

$f(t)=\lambda_{2} \sum_{\tau=1}^{t} \tau^{2}+\lambda_{3} \sum_{\tau=1}^{t} \tau+\lambda_{4}$

The details of the TDPFOGM can be found in (Chen et al., 2018). The predicted values of the original series can be obtained using the fractional-order inverse accumulative generation operation as follows:

$\hat{x}^{(0)}=\hat{x}^{\left(\frac{p}{q}\right)\left(\frac{p}{q}\right)}(k)=\left\{x^{\left(\frac{p}{q}\right)\left(\frac{p}{q}\right)}(1), x^{\left(\frac{p}{q}\right)\left(\frac{p}{q}\right)}(2), \ldots, x^{\left(\frac{p}{q}\right)\left(\frac{p}{q}\right)}(n)\right\}$

\subsection{Quasi-Newton Non-Linear Optimization Technique for Gas Lift Optimization}

The quasi-Newton non-linear optimization technique incorporated with the gradient projection method was used for gas lift optimization (Namdar, 2019). This method is capable of accommodating restrictions to gas injection rates. The only requirement for fast convergence is that a reasonable estimate of the gas injection rates must be supplied as an initial point to the optimization method.

\subsection{Newton Reduction Method Approach for Gas Lift Optimization}

The Newton reduction method, which is based on an upper convex profile requirement, reduces a high dimensional problem into one of the single variables. This method is designed for the treatment of the gas lift optimization problem, and it is applied by enforcing the notion of good separability and the availability of upper convex lift profiles for each well (Rashid, 2010). Because of interdependent wells, the procedure is performed iteratively until convergence on wellhead pressures.

\subsection{Non-Linear Programming Approach to Gas Lift Optimization}

Gas lift allocation can be modeled using a non-linear programming approach. This can be achieved by adjusting the optimum gas injection rates and compressor pressure to maximize the oil production rate or other objective functions (Khishvand \& Khamehchi, 2012). The non-linear 
programming approach was deployed for gas lift optimization (Codas et al., 2016; Namdar, 2019; Namdar \& Shahmohammadi, 2019; Sharma et al., 2012).

\subsection{Feedback Control Structures in The Gas Lift}

Production increases can be achieved in gas lift wells by stabilizing the casing-heading (Hansen, 2012). Optimal operation in the Oil and gas wells can be achieved using simple feedback control structures (Krishnamoorthy et al., 2019). Feedback control structures can also reduce operational problems when implemented in the gas lift wells. The use of feedback control has the advantage over the traditional methods in limiting the production losses associated with feedback control stabilization.

\section{Conclusion}

Artificial gas injection into aging wells enhances reservoir pressures and boosts production rates. The gas lift process involves injecting gas through the tubing-casing annulus. The injected gas aerates the fluid to reduce its density; the pressure is then able to lift the oil column and forces the liquid out of the wellbore. The amount of gas to be injected to maximize oil production varies based on well geometries and conditions. Gas lift optimizations can be achieved using Machine Learning and Artificial intelligence techniques. Most of the algorithms used in the optimization strategies in this review are metaheuristic algorithms. A right lift optimization can reduce the operating cost, increase the net present value (NPV), and maximize the recovery from the asset.

\section{References}

Abdalsadig, M. ., Nourian, A., Nasr, G. ., \& Babaie, M. (2016). Gas lift optimization to improve well performance. http://usir.salford.ac.uk/38144/

Bordbar, M., Neshat, A., Javadi, S., Pradhan, B., \& Aghamohammadi, H. (2020). Meta-heuristic algorithms in optimizing GALDIT framework: A comparative study for coastal aquifer vulnerability assessment. Journal of Hydrology, 585, 124768. https://doi.org/10.1016/j.jhydrol.2020.124768

Chen, L., Liu, Z., \& Ma, N. (2018). Time-Delayed Polynomial Grey System Model with the Fractional Order Accumulation. Mathematical Problems in Engineering, 2018. https://doi.org/10.1155/2018/3640625

Chen, L., Liu, Z., \& Ma, N. (2019). Optimize production allocation for the oil-gas field basing on a novel grey model. Journal of Natural Gas Geoscience, 4(2), 121-128. https://doi.org/10.1016/j.jnggs.2019.03.003

Christiansen, L. H., Hørsholt, S., \& Jørgensen, J. B. (2018). A Least Squares Method for Ensemble-based Multi-objective Oil Production Optimization. IFAC-PapersOnLine, 51(8), 712. https://doi.org/10.1016/j.ifacol.2018.06.347

Codas, A., Jahanshahi, E., \& Foss, B. (2016). A two-layer structure for stabilization and optimization of an oil gathering network. IFAC-PapersOnLine, 49(7), 931-936. https://doi.org/10.1016/j.ifacol.2016.07.317

Duran Toksarı, M. (2007). Ant colony optimization approach to estimate energy demand of Turkey. Energy Policy, 35(8), 3984-3990. https://doi.org/10.1016/j.enpol.2007.01.028 
François, G., \& Bonvin, D. (2014). Use of transient measurements for the optimization of steadystate performance via modifier adaptation. Industrial and Engineering Chemistry Research, 53(13), 5148-5159. https://doi.org/10.1021/ie401392s

Ghaedi, M., Ghotbi, C., \& Aminshahidy, B. (2014). The optimization of gas allocation to a group of wells in a gas lift using an efficient Ant Colony Algorithm (ACO). Energy Sources, Part A: Recovery, Utilization and Environmental Effects, 36(11), 1234-1248. https://doi.org/10.1080/15567036.2010.536829

Hamedi, H., Rashidi, F., \& Khamehchi, E. (2011). A novel approach to the gas-lift allocation optimization problem. Petroleum Science and Technology, 29(4), 418-427. https://doi.org/10.1080/10916460903394110

Hansen, H. H. (2012). A Comparative Study of Control Structures Applied in Gas Lift Systems to Prevent Casing Heading [Norwegian University of Science and Technology]. http://folk.ntnu.no/skoge/diplom/diplom12/hansen/Diplom_Henrik_Hansen_DAIM.pdf

Hsu, C.-C., \& Chen, C.-Y. (2003). Applications of improved grey prediction model for power demand forecasting. Energy Conversion and Management, 44(14), 2241-2249. https://doi.org/10.1016/S0196-8904(02)00248-0

Ismail, W. R. B., \& Trjangganung, K. (2014). Mature field gas lift optimisation: Challenges \& strategies, case study of D-field, Malaysia. Society of Petroleum Engineers - International Petroleum Technology Conference 2014, IPTC 2014 - Innovation and Collaboration: Keys to Affordable Energy, 2, 1703-1714. https://doi.org/10.2523/iptc-17896-ms

Kadkhodaie-llkhchi, A. (2015). A systematic approach for estimation of reservoir rock properties using Ant Colony Optimization. Geopersia, 5(1), 7-17.

Kesavan, V., Kamalakannan, R., Sudhakarapandian, R., \& Sivakumar, P. (2020). Heuristic and meta-heuristic algorithms for solving medium and large scale sized cellular manufacturing system NP-hard problems: A comprehensive review. Materials Today: Proceedings, 21, 6672. https://doi.org/10.1016/j.matpr.2019.05.363

Khishvand, M., \& Khamehchi, E. (2012). Nonlinear risk optimization approach to gas lift allocation optimization. Industrial and Engineering Chemistry Research, 51(6), 2637-2643. https://doi.org/10.1021/ie201336a

Krishnamoorthy, D., Fjalestad, K., \& Skogestad, S. (2019). Optimal operation of oil and gas production using simple feedback control structures. Control Engineering Practice, 91, 104107. https://doi.org/10.1016/j.conengprac.2019.104107

Krishnamoorthy, D., Jahanshahi, E., \& Skogestad, S. (2018). Gas-lift Optimization by Controlling Marginal Gas-Oil Ratio using Transient Measurements*. IFAC-PapersOnLine, 51(8), 19-24. https://doi.org/10.1016/j.ifacol.2018.06.349

Kumar, A., Soota, T., \& Kumar, J. (2018). Optimisation of wire-cut EDM process parameter by Grey-based response surface methodology. Journal of Industrial Engineering International, 14(4), 821-829. https://doi.org/10.1007/s40092-018-0264-8

López, S., Koç, U., Bakker, E., \& Rahmani, J. (2019). Optimization of Lift Gas Allocation using Evolutionary Algorithms. International Journal of Computer Applications Technology and Research, 8(9), 353-357. https://doi.org/10.7753/ijcatr0809.1003 
Ma, H., \& Wu, Y. (2010). A Grey Forecasting Model Based on BP Neural Network for Crude Oil Production and Consumption in China. 2010 Third International Symposium on Information Processing, 105-109. https://doi.org/10.1109/ISIP.2010.12

Mahdiani, M. R., \& Khamehchi, E. (2015). Preventing Instability Phenomenon in Gas-lift Optimization. In Iranian Journal of Oil \& Gas Science and Technology (Vol. 4, Issue 1). http://ijogst.put.ac.ir

Namdar, H. (2019). Developing an improved approach to solving a new gas lift optimization problem. Journal of Petroleum Exploration and Production Technology. https://doi.org/10.1007/s13202-019-0697-7

Namdar, H., \& Shahmohammadi, M. A. (2019, November 2). Optimization of production and liftgas allocation to producing wells by a new developed GLPC correlation and a simple optimization method. Energy Sources, Part A: Recovery, Utilization and Environmental Effects. https://doi.org/10.1080/15567036.2019.1568635

Nishikiori, N., Redner, R. A., Doty, D. R., \& Schmidt, Z. (1989, April 4). An Improved Method for Gas Lift Allocation Optimization. SPE Annual Technical Conference and Exhibition. https://doi.org/10.2118/19711-MS

Oloro, J., \& Ogbolu, E. (2013). Gas Lift Optimization: Using Least Square Method and Solver. Greener Journal of Petroleum and Gas Exploration, 1(1), 1-006. www.gjournals.org

Rashid, K. (2010). Optimal allocation procedure for gas-lift optimization. Industrial and Engineering Chemistry Research, 49(5), 2286-2294. https://doi.org/10.1021/ie900867r

Rashid, K., Bailey, W., \& Couët, B. B. (2012). A Survey of Methods for Gas-Lift Optimization. Modelling and Simulation in Engineering, 2012, 16. https://doi.org/10.1155/2012/516807

Saepudin, D., Soewono, E., Sidarto, K. A., Gunawan, A. Y., Siregar, S., \& Sukarno, P. (2007). An investigation on gas lift performance curve in an oil-producing well. International Journal of Mathematics and Mathematical Sciences, 2007. https://doi.org/10.1155/2007/81519

Sharma, R., Fjalestad, K., \& Glemmestad, B. (2012). Optimization of lift gas allocation in a gas lifted oil field as non-linear optimization problem. Modeling, Identification and Control, 33(1), 13-25. https://doi.org/10.4173/mic.2012.1.2

Sukarno, P., Saepudin, D., Dewi, S., Soewono, E., Sidarto, K. A., \& Gunawan, A. Y. (2009). Optimization of gas injection allocation in a dual gas lift well system. Journal of Energy Resources Technology, Transactions of the ASME, 131(3), 0331011-0331017. https://doi.org/10.1115/1.3185345

Sylvester, O. (2015). Gas Lift Technique a Tool to Production Optimization. International Journal of Oil, Gas and Coal Engineering, 3(3), 41. https://doi.org/10.11648/j.ogce.20150303.12

Tavakoli, R., Daryasafar, A., Mohammad, K., \& Milad, B. (2017). Optimization of Gas Lift Allocation Using Different Models. Recent Advances in Petrochemical Science, 1(2). https://doi.org/10.19080/rapsci.2017.01.555559

Truong, D. Q., \& Ahn, K. K. (2012). An accurate signal estimator using a novel smart adaptive grey model SAGM(1,1). Expert Systems with Applications, 39(9), 7611-7620. https://doi.org/10.1016/j.eswa.2012.01.002 
Vijayalakshmi, P., Francis, S. A. J., \& Dinakaran, J. A. (2015). A robust energy efficient ant colony optimization routing algorithm for multi-hop ad hoc networks in MANETs. Wireless Networks 2015 22:6, 22(6), 2081-2100. https://doi.org/10.1007/S11276-015-1082-1

Yakoot, M. S., Shedid, S. A., \& Arafa, M. I. (2014). A simulation approach for optimization of gas lift performance and multi-well networking in an Egyptian oil field. Proceedings of the Annual Offshore Technology Conference. https://doi.org/10.4043/24703-ms

Zerafat, M. M., Ayatollahi, S., \& Roosta, A. A. (2009). Genetic Algorithms and Ant Colony Approach for Gas-lift Allocation Optimization. Journal of the Japan Petroleum Institute, 52(3), 102-107. https://doi.org/10.1627/jpi.52.102. 